\section{Zur Rechtswidrigkeit der Ausschreibung von Kontrastmitteln durch die Krankenkassen und der Bezugsverpflichtung für Radiologen}

\section{Einleitung}

Die Anwendung von Kontrastmitteln durch Radiologen im Rahmen der Schnittbilddiagnostik stellt einen nicht unerheblichen Kostenfaktor im Rahmen der vertragsärztlichen Versorgung dar. Die Krankenkassen versuchen deshalb seit jeher, Preissenkungen zu erzielen. Mit der Presseberichterstattung aus dem vergangenen Jahr ist das Thema der Kontrastmittelabrechnung in den Fokus einer breiten Öffentlichkeit gerückt worden. ${ }^{1}$ Radiologen sollen Kontrastmittel für CT- oder MRT-Untersuchungen günstig bei den Herstellern eingekauft und den Krankenkassen im Rahmen von Erstattungspauschalen weit höhere Preise in Rechnung gestellt haben. Die Bundesregierung und der Berufsverband der Deutschen Radiologen (BDR) haben zwar darauf hingewiesen, dass die Erstattungspauschalen regelmäßig angepasst werden und hohe Gewinne nur temporär anfallen. ${ }^{2}$ Dennoch haben sich die Krankenkassen nach der Debatte um die Preise von Kontrastmitteln in den Bezirken mehrerer Kassenärztlicher Vereinigungen (KV) für neue Regelungen entschieden.
Neben der Kürzung der Kontrastmittelpauschalen auf ein zum Teil nicht mehr kostendeckendes Niveau, wie sie beispielsweise in Bayern vorgenommen wurde, führen die Krankenkassen in anderen KV-Bezirken vermehrt wirkstoffübergreifende Ausschreibungsverfahren für Lieferverträge mit Pharmaunternehmen über die Belieferung mit Kontrastmitteln durch. Die niedergelassenen Radiologen sollen dann unter Berufung auf das im Vertragsarztrecht geltende Wirtschaftlichkeitsgebot (vgl. §§2 Abs. 1, 12 Abs. 1, 70 Abs. 1 SGB V), in dessen Rahmen gemäß § 9 Abs. 2 Nr. 2 Arzneimittel-Richtlinie $(A M-R L)^{3}$ das Minimalprinzip ${ }^{4}$ anzuwenden sei, und mit der Ankündigung sowie Durchführung von Regressverfahren zu einem Bezug bestimmter Kontrastmittel bei bestimmten Unternehmen verpflichtet werden.

Bereits im Jahr 2018 hat das Sozialgericht (SG) Düsseldorf solche Exklusivlieferverträge als für mit den Vorschriften des SGB V nicht vereinbar gehalten. ${ }^{5}$ Die Kassenärztliche Bundesvereinigung (KBV), verschiedene KVen und auch der BDR haben sich stets gegen die wirkstoffübergreifende Ausschreibung mit Bezugsverpflichtung für die

3 Kontrastmittel sind nach § 2 Abs. 1 Nr. 2 Arzneimittelgesetz (AMG) Arzneimittel.

4 Das Minimalprinzip bedingt nach der Rechtsprechung des Bundessozialgerichts (BSG), „dass bei Existenz verschiedener gleich zweckmäßiger und notwendiger Behandlungsmöglichkeiten die Kosten für den gleichen zu erwartenden Erfolg geringer oder zumindest nicht höher sind“. Das gilt „auch im Verhältnis zweier therapeutisch gleichwertiger, aber unterschiedlich teurer Arzneimittel“ (BSG, Urteil vom 17.02.2016, Az.: B 6 KA 3/15R= juris).

5 SG Düsseldorf, Urteil vom 29. November 2018, Az.: S 8 KR 219/18 = juris.

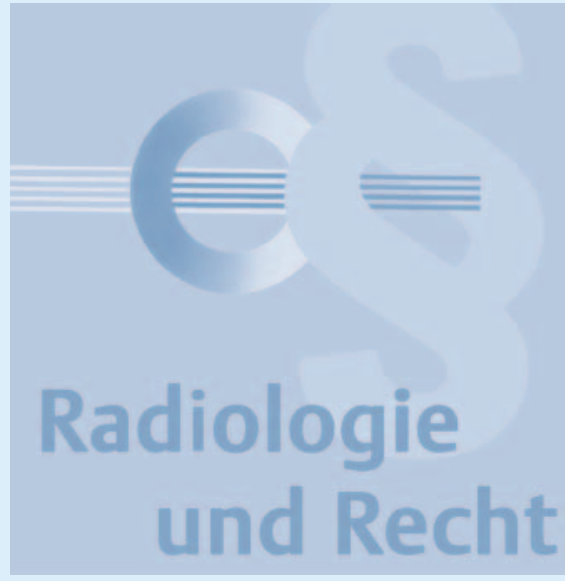

niedergelassenen Radiologen ausgesprochen. ${ }^{6}$ Gleichwohl erfolgen weiterhin Ausschreibungen durch die Krankenkassen und Regressverfahren gegen die niedergelassenen Radiologen in einem zum Teil existenzbedrohenden Ausmaß.

In dem folgenden Beitrag stellen wir deshalb die Gründe dar, aus denen die Ausschreibung von Kontrastmitteln in Verbindung mit einer Bezugsverpflichtung für die Radiologen und damit auch die Festsetzung von Regressen gegen die Radiologen rechtswidrig sind.

\section{Das Urteil des SG Düsseldorf}

Der Entscheidung des SG Düsseldorf lag zwar kein Regressverfahren gegen eine radiologische Praxis zugrunde, jedoch war

6 Entwurf eines Gesetzes für mehr Sicherheit in der Arzneimittelversorgung - Stellungnahme der KBV zum Gesetzentwurf der Bundesregierung vom 27. März 2019 (BT-Drucksache 19/8753), abrufbar unter: https://www.kbv. de/media/sp/2019_04_09_GSAV_KBV_ Stellungnahme_Regierungsentwurf.pdf (zuletzt abgerufen am 08.07.2020); Statement der Kassenärztlichen Vereinigung Nordrhein zur wirkstoffübergreifenden Ausschreibung Röntgenkontrastmittel, abrufbar unter: https://www.kvno.de/downloads/verordnun gen/statement_roentgenkontrastmittel.pdf (zuletzt abgerufen am 08.07.2020); Stellungnahme zum Entwurf des Terminservice- und Versorgungsgesetzes des Berufsverbands der Deutschen Radiologen, abrufbar unter: https://www.bundesgesundheitsministerium. de/fileadmin/Dateien/3_Downloads/ Gesetze_und_Verordnungen/Stellungnah men_WP19/TSVG/TSVG_BDR2.pdf (zuletzt abgerufen am 08.07.2020). 
der Streit zwischen einem Pharmaunternehmen und einer Krankenkasse um die Vergütung von Kontrastmittellieferungen an radiologische Praxen zu entscheiden. Das Pharmaunternehmen hatte radiologische Praxen aufgrund deren Verordnungen mit Kontrastmitteln im Bereich der KV Rheinland-Pfalz beliefert. Die Vergütung des Pharmaunternehmens sollte unmittelbar durch die Krankenkasse erfolgen.

Die Krankenkasse lehnte die Zahlung jedoch mit dem Verweis auf die von ihr durchgeführten Ausschreibungen und die ausschreibungsgewinnenden Unternehmen sowie Kontrastmittel ab. Aufgrund des im Vertragsarztrecht geltenden Wirtschaftlichkeitsgebots seien die radiologischen Praxen nicht berechtigt gewesen, die Kontrastmittel bei dem klagenden Pharmaunternehmen zu bestellen und dieses sei nicht berechtigt gewesen, die radiologischen Praxen mit Kontrastmitteln zu beliefern. Eine Vergütung stehe dem Pharmaunternehmen daher gegen die Krankenkasse nicht zu.

Das Sozialgericht entschied zugunsten des Pharmaunternehmens, dass die Ausschreibung und der Abschluss von Exklusivverträgen einer gesetzlichen Ermächtigungsgrundlage bedurft hätten, die jedoch nicht vorhanden sei. §130a SGB V, der die Voraussetzungen für Rabatte auf Arzneimittel in der gesetzlichen Krankenversicherung regelt, sehe im Verhältnis zu Pharmaunternehmen allein eine Gestaltung mittels Rabattverträgen und Festbeträgen vor. §130a Abs. 8 S. 9 SGB V7 schreibe insoweit ausdrücklich vor, dass der Vielfalt der Anbieter bei Abschluss der Rabattverträge Rechnung zu tragen sei. Wörtlich führt das SG Düsseldorf weiter aus:

Bereits gegen diese Regelungsvorgabe zur Umsetzung des Wirtschaftlichkeitsgebotes bei der Versorgung mit Arzneimitteln verstoßen die von der Beklagten geschlossenen Exklusivlieferverträge. [...] Das Instrument einer
Ausschreibung mit Exklusivverträgen hat der Gesetzgeber nur im Hilfsmittelbereich vorgesehen (§ 127 SGB V), dagegen nicht im Arzneimittelbereich.

Die von der Krankenkasse durchgeführte Ausschreibung, verbunden mit dem Abschluss von Exklusivverträgen mit anderen Pharmaunternehmen über die Belieferung mit Kontrastmitteln sei demnach, aufgrund der fehlenden Ermächtigungsgrundlage, rechtswidrig gewesen.

Der Rechtsstreit ist inzwischen in der Berufungsinstanz beim Landessozialgericht (LSG) Nordrhein-Westfalen anhängig. ${ }^{8}$

\section{Rechtswidrigkeit der Bezugsverpflichtung}

Den niedergelassenen Radiologen den Bezug bestimmter Kontrastmittel bei bestimmten Unternehmen vorzuschreiben ist zudem aus den nachfolgenden Gründen rechtswidrig.

\section{Keine ausreichende Rechtsgrundlage}

Die Krankenkassen berufen sich, um den Radiologen den Bezug bestimmter Kontrastmittel bei bestimmten Unternehmen vorzuschreiben, stets auf das im Vertragsarztrecht geltende Wirtschaftlichkeitsgebot. Aufgrund des in dessen Rahmen anzuwendenden Minimalprinzips seien die Radiologen verpflichtet, bei dem Bezug von Kontrastmitteln den wirtschaftlichsten Bezugsweg einzuhalten und das wirtschaftlichste Produkt zu wählen.

Aufgrund der von den Krankenkassen durchgeführten Ausschreibungsverfahren seien dies die ausschreibungsgewinnenden Unternehmen und Kontrastmittel; ggf. sei das von den Radiologen bislang verordnete Kontrastmittel wirkstoffübergreifend durch das bezuschlagte Produkt zu substituieren (Aut-simile-Substitution).
Ungeachtet der Frage, ob dies vergaberechtlich zulässig ist, ${ }^{9}$ existiert aus den Vorschriften des Sozialrechts jedoch keine Rechtsgrundlage dafür, einem Vertragsarzt eine sogar wirkstoffübergreifende Substitution von Arzneimitteln vorzuschreiben.

Wie das BSG betont, ${ }^{10}$ ist es

nur in besonders gelagerten Konstellationen möglich, unmittelbar aus dem Wirtschaftlichkeitsgebot konkrete, im Falle der Nichtbeachtung einen Regress auslösende Vorgaben für die ärztliche Behandlung eines Patienten im Einzelfall abzuleiten.

Dabei bedürfe es, so das BSG weiter,

regelmäßig näherer normativer Konkretisierungen - etwa hinsichtlich der Verordnungsfähigkeit von bestimmten Arzneimitteln -, an denen der Arzt seine Behandlungsweise ausrichten kann. Dies ergibt sich bereits daraus, dass der GBA gemäß §92 Abs 1 Satz 2 Nr 6 $S G B V$ gehalten ist, eine Richtlinie für die Verordnung von Arzneimitteln zu beschließen, welche den in § 92 Abs 2 SGB V niedergelegten detaillierten Vorgaben genügen müssen; hierzu gehören auch Regelungen, die dem Vertragsarzt eine Entscheidung über die Wirtschaftlichkeit einer Verordnung ermöglichen.

Die Regelungen über die Rabattverträge nach §130a Abs. 8 SGB V sind nicht anwendbar und enthalten zudem keine Rechtsgrundlage, um den Radiologen eine Bezugsverpflichtung für Kontrastmittel aufzuerlegen. Nach dem Wortlaut des $§ 129$ Abs. 1 S. 3 SGB $V$ sind im Rahmen von

9 Die Vergabekammern hatten die Ausschreibungen nach der Vergabe- und Vertragsordnung für Leistungen und dem Gesetz gegen Wettbewerbsbeschränkungen überwiegend als zulässig angesehen (vgl. BKartA (2. Vergabekammer des Bundes), Beschluss vom 20.04.2009, Az.: VK 2-36/09= juris und BKartA (3. Vergabekammer des Bundes), Beschluss vom 20.01.2009, Az.: VK 3-191/ $08=$ juris). Das SG Düsseldorf weist in seinem Urteil vom 29.11.2018 jedoch daraufhin, dass die Vergabekammern „lediglich die Art und Weise der Durchführung des Vergabeverfahrens“, nicht jedoch überprüfen könnten, „ob ein Vergabeverfahren nach den einschlägigen Vorschriften für die gesetzliche Krankenversicherung zulässig war“.

10 BSG, Urteil vom 13.05.2015, Az.: B 6 KA 18/ $14 \mathrm{R}=$ juris. 
Rabattverträgen lediglich Apotheken bei der Abgabe verordneter Arzneimittel an Versicherte zur Beachtung der zwischen den Krankenkassen und den Herstellern abgeschlossenen Verträgen verpflichtet. Dabei kann der Vertragsarzt jedoch nach $\S 73$ Abs. 5 S. 2 SGB V „ausschließen, dass die Apotheken ein preisgünstigeres wirkstoffgleiches Arzneimittel anstelle des verordneten Mittels abgeben“ (sog. Aut-idem-Regelung). Ein entsprechendes Reglement besteht für die Substitution von Kontrastmitteln, die als Sprechstundenbedarf nach den Sprechstundenbedarfsvereinbarungen oder außerhalb dieser verordnet werden, im SGB V nicht.

Die Landesverbände der Krankenkassen und der Ersatzkassen sind mit den KVen gemäß § 82 Abs. 2 S. 1 SGB V lediglich gemeinsam berechtigt, die Vergütungen der an der vertragsärztlichen Versorgung teilnehmenden Ärzte und ärztlich geleiteten Einrichtungen durch Gesamtvertrag zu regeln. Hierunter fällt auch die Berechtigung zum Erlass der Sprechstundenbedarfsvereinbarung oder einer „Kontrastmittelvereinbarung “ über die Verordnung und die Preise von Kontrastmitteln. ${ }^{11}$ Die Vergütungsregelungen sind jedoch gemeinsam und einheitlich mit den KVen im Rahmen der gesamtvertraglichen Normsetzungskompetenz zu vereinbaren. Darüber hinaus wäre von dem normativen Gestaltungsspielraum der gesamtvertraglichen $\mathrm{Er}$ mächtigung in $\S 82$ Abs. 2 S. 1 SGB V aktuell keine Vergütungsregelung gedeckt, die ein Ausschreibungsverfahren und eine Bezugsverpflichtung von Kontrastmitteln gegenüber den Radiologen vorsieht. Aufgrund des damit verbundenen weitreichenden Eingriffs in die Therapiefreiheit der Ärzte, bedürfte eine solche Vereinbarung einer ausdrückliche Regelung im SGB V. Die Krankenkassen haben im Rahmen der von ihnen durchgeführten Ausschreibungsverfahren und der statuierten Bezugsverpflichtung bisher jedoch nicht im Rahmen der gesamtvertraglichen Normsetzungskompetenz nach $\S 82$ Abs. 2 S. 1 SGB V, sondern alleine gehandelt. Zudem sieht $\S 82$ Abs. 2 S. 1 SGB V keine entsprechende Regelungskompetenz des Ausschreibungsverfahrens und der Rechtsfolgen vor.

11 Vgl. SG München, Beschluss vom 09.08.2001, Az.: S 33 KA 2031/01 ER = NZS 2002, 50, 51.
Den Radiologen kann demnach von den Krankenkassen nicht vorgeschrieben werden, ausschließlich ein bezuschlagtes Kontrastmittel zu verordnen. Dies beruht weiterhin darauf, dass §47 AMG nach der Rechtsprechung des BSG keine Ausnahme von der Apothekenpflicht des §43 AMG vorsieht, sondern lediglich die Befugnis der Pharmaunternehmen und Großhändler normiert, die apothekenpflichtigen Arzneimittel an bestimmte Personen und Institutionen außerhalb des Apothekenbereichs abzugeben. ${ }^{12}$ Das wird auch durch die Regelung in $\S 40$ Abs. 3 S. 1 AM-RL bestätigt, in der der Gemeinsame Bundesausschuss (G-BA) Hinweise zur Austauschbarkeit von Darreichungsformen (aut idem) gegeben hat:

(3) Die Möglichkeit der verordnenden Ärztin oder des verordnenden Arztes, unter Würdigung patientenindividueller und erkrankungsspezifischer Aspekte nach § 73 Absatz 5 Satz 2 SGB V die Ersetzung durch ein wirkstoffgleiches Arzneimittel auszuschließen, bleibt von diesen Regelungen unberührt.

Dass §130a SGB keine ausreichende Rechtsgrundlage darstellt, um den Radiologen den Bezug bestimmter Kontrastmittel bei bestimmten Unternehmen vorzuschreiben, wird darüber hinaus dadurch offenbar, dass die vermeintlich bindende Bestimmung, welche Arzneimittel und Wirkstoffe austauschbar sein sollen, durch die ausschreibenden Krankenkassen erfolgt, ohne dass die Kriterien hierfür offengelegt würden. Selbst soweit eine wirkstoffgleiche Austauschbarkeit von Arzneimitteln gesetzlich vorgesehen ist, ordnet das Gesetz in $\S 129$ SGB V und den Rahmenverträgen die Voraussetzungen hierfür aber selbst an oder delegiert die Bestimmung der Kriterien an den G-BA; es überlässt sie aber selbstverständlich nicht den Krankenkassen. ${ }^{13}$ Dass aufgrund einer Einschätzung

12 Vgl. BSG, Urteil vom 27.10.2009, Az.: B 1 KR $7 / 09 R=$ juris; BSG, Urteil vom 29.04.2010, Az.: B 3 KR 3/09 R= juris; BSG, Urteil vom 13.05.2015, Az:: B 6 KA 18/14R= juris.

13 Zur Notwendigkeit der Feststellung der Austauschbarkeit aus haftungsrechtlichen Gründen durch den G-BA vgl. die Neuregelung zu Biosimilars, Gesetzesbegründung zum Gesetz für mehr Sicherheit in der Arzneimittelversorgung - GSAV, BT-Drs. 19/8753 vom 27.03.2019, S. $89 \mathrm{f}$ der in diesem Zusammenhang vornehmlich wirtschaftliche Interessen verfolgenden Krankenkassen eine Substitution von wirkstoffverschiedenen Arzneimitteln erfolgen soll, entbehrt demgegenüber einer gesetzlichen Grundlage.

Durch die verbindliche Vorgabe der Substitution durch die Krankenkassen würde schließlich erheblich in die Therapiefreiheit der verordnenden Radiologen eingegriffen. Zwar gilt die Therapiefreiheit des Arztes im Gesamtsystem des Vertragsarztrechts nicht unbeschränkt und ist z. B. mit dem Wirtschaftlichkeitsgebot abzuwägen. ${ }^{14}$ Gleichzeitig kann es aber nicht zulässig sein, dass der verordnende Radiologe das Haftungsrisiko gegenüber den Patienten für die Verwendung des bezuschlagten, ggf. wirkstoffverschiedenen und vermeintlich von ihm zu verwendenden Kontrastmittels zu tragen hat. Dies gilt insbesondere vor dem Hintergrund der von der KBV hervorgehobenen haftungsrechtlichen Fragen bei der wirkstoffübergreifenden Substitution von Kontrastmitteln, die sich insbesondere bei gadoliniumhaltigen Kontrastmitteln stellen. ${ }^{15}$

Das Vorgehen der Krankenkassen verstößt insoweit gegen den nach $\S 31$ SGB I auch für die Krankenkassen geltenden Gesetzesvorbehalt, wonach Rechte und Pflichten in den Sozialleistungsbereichen nur begründet, festgestellt, geändert oder aufgehoben werden dürfen, soweit ein Gesetz dies vorschreibt oder zulässt.

\section{Kein Verstoß gegen das Wirtschaftlichkeitsgebot}

Selbst wenn aber das Wirtschaftlichkeitsgebot grundsätzlich eine ausreichende Rechtsgrundlage wäre, um den Radiologen den Bezug bestimmter Kontrastmittel bei

14 BSG, Urteil vom 08.09.1993, Az.: 14a RKa 7/ 92 = juris; BSG, Urteil vom 20.03.1996, Az.: 6 RKa 62/94 = juris; BSG, Urteil vom 25.09.2000, Az.: B 1 KR 24/99R=juris.

15 Vgl. Entwurf eines Gesetzes für mehr Sicherheit in der Arzneimittelversorgung - Stellungnahme der KBV zum Gesetzentwurf der Bundesregierung vom 27. März 2019 (BT-Drucksache 19/8753), S. 14, abrufbar unter: https://www.kbv.de/media/sp/ 2019_04_09_GSAV_KBV_Stellungnahme_ Regierungsentwurf.pdf (zuletzt abgerufen am 08.07.2020). 
bestimmten Unternehmen vorzuschreiben, würde eine von den Vorgaben der Krankenkassen abweichende Verordnung durch die Radiologen nicht gegen das Wirtschaftlichkeitsgebot in der Ausprägung des Minimalprinzips verstoßen.

Voraussetzung für einen Verstoß gegen das Wirtschaftlichkeitsgebot wäre nämlich, dass diejenigen Kontrastmittel, die die Radiologen mit den von den Krankenkassen bezuschlagten Kontrastmitteln substituieren sollen, mit letzteren therapeutisch gleichwertig sind. Nach einem Urteil des LSG Berlin-Brandenburg, ${ }^{16}$ auf das vom BSG verwiesen wird, ${ }^{17}$ ist bei zwei in Betracht kommenden Arzneimitteln „die therapeutische Gleichwertigkeit beider Arzneimittel durch einen auf den betroffenen Versicherten bezogenen Vergleich möglicher Nebenwirkungen sowie ggf. pharmakodynamischer und -kinetischer Eigenschaften zu klären“. Erst auf der Grundlage einer solchen Konkretisierung sind die Frage nach der therapeutischen Gleichwertigkeit und - darauf aufbauend - ein Kostenvergleich möglich.

Von den Krankenkassen wird ein solcher Vergleich im Rahmen der Ausschreibungen und der Anordnungen der Bezugsverpflichtungen an die Radiologen zumindest nicht nachvollziehbar und transparent vorgenommen und veröffentlicht. Im Rahmen der Ausschreibungen durch die Krankenkassen ist eine versichertenbezogene Prüfung ohnehin nicht möglich. Dies gilt auch im Rahmen der Verordnung der Kontrastmittel als Sprechstundenbedarf. Denn der Sprechstundenbedarf wird gerade nicht versichertenbezogen verordnet.

Erst wenn ein solcher Vergleich der Kontrastmittel ergeben würde, dass diese als therapeutisch gleichwertig anzusehen sind, wäre in einem weiteren Schritt zu ermitteln, ob zumindest eines dieser Alternativpräparate preiswerter ist. ${ }^{18}$ Erfolgt eine solche Feststellung bzgl. der Vergleichbarkeit

16 LSG Berlin-Brandenburg, Urteil vom 28.10.2009, Az.: L 7 KA 131/06 = juris.

17 BSG, Urteil vom 17.02.2016, Az.: B 6 KA 3/ $15 R=$ juris.

18 Vgl. LSG Berlin-Brandenburg, Urteil vom 28.10.2009, Az.: L 7 KA 131/06 = juris. der Arzneimittel und die darauf beruhende Ermittlung, dass das Alternativpräparat tatsächlich preiswerter ist, weder im Rahmen der Ausschreibungen durch die Krankenkassen noch im Rahmen der Verordnungsprüfung, kann eine Bezugsverpflichtung für die Radiologen nicht mit dem Wirtschaftlichkeitsgebot begründet werden.

\section{Fazit}

Nach dem Vorstehenden sind die Ausschreibung von Kontrastmitteln durch die Krankenkassen und die Anordnung einer Bezugsverpflichtung an die Radiologen rechtswidrig. Eine Rechtsgrundlage besteht weder dafür, den Radiologen einen bestimmten Bezugsweg noch ihnen die wirkstoffübergreifende Substitution von Kontrastmitteln vorzuschreiben. Auch mit dem Wirtschaftlichkeitsgebot lässt sich dies nicht begründen. Auf der Grundlage des Wirtschaftlichkeitsgebots dürfte den Radiologen aber ohnedies, ohne einen ausreichenden Vergleich der therapeutischen Gleichwertigkeit verschiedener Kontrastmittel, deren Substitution nicht vorgeschrieben werden.

Diese Auffassung ist durch die Rechtsprechung allerdings noch nicht abschließend bestätigt worden. In denjenigen KV-Bezirken, in denen durch die Krankenkassen Ausschreibungen für Kontrastmittel durchgeführt worden sind, wird deshalb weiterhin zu besonderer Aufmerksamkeit und dazu geraten - soweit dies medizinisch vertretbar ist - möglichst auf das von den Krankenkassen bezuschlagte Kontrastmittel auszuweichen und dieses bei dem bezuschlagten Unternehmen zu beziehen. Soweit die Substitution aus medizinischen Gründen nicht vertretbar ist, sollten die Gründe für die von den Vorgaben der Krankenkassen abweichende Verordnung möglichst genau in der Behandlungsdokumentation erfasst und die Verordnungen, auch wenn hierzu ebenfalls keine Rechtsgrundlage besteht, ${ }^{19}$

19 Eine medizinische Begründung auf dem Verordnungsblatt ist weder im Bundesmantelvertrag-Ärzte noch in der Vordruckvereinbarung über Vordrucke für die vertragsärztliche Versorgung vorgesehen. entsprechend begründet werden. arallel sollte im Einzelfall geprüft werden, ob gegen die entsprechenden Honorarbescheide oder Abrechnungen Widerspruch eingelegt und inzident oder, soweit dies verfahrensrechtlich nicht möglich ist, im Rahmen einer Feststellungsklage die Rechtmäßigkeit der Ausschreibungen und die Bezugsverpflichtung geprüft werden können.

Soweit von den Vorgaben der Krankenkassen abweichende Verordnungen von Kontrastmitteln vorgenommen worden sind, müssen von den Prüfungseinrichtungen festgesetzte Regresse ebenfalls nicht widerspruchslos hingenommen werden. Wie dargestellt sprechen erhebliche Gründe für die Rechtswidrigkeit der Ausschreibung von Kontrastmitteln durch die Krankenkassen und die Anordnung der Bezugsverpflichtung gegenüber den Radiologen. Widersprüche und Klagen gegen entsprechende Regressforderungen sind insoweit nicht aussichtslos und zudem geeignet, künftig im Zusammenhang mit der Kontrastmittelverordnung zu einer größeren Rechtssicherheit für betroffene Radiologen beizutragen.

Prof. Dr. Peter Wigge

Rechtsanwalt

Fachanwalt für Medizinrecht

Jonas Kaufhold

Rechtsanwalt

Rechtsanwälte Wigge

Scharnhorststraße 40

48151 Münster

Telefon: (0251) 53 595-0

Telefax: (0251) 53 595-99

E-Mail: kanzlei@ra-wigge.de

www.ra-wigge.de 